ARTIGO ORIGINAL ORIGINAL ARTICLE

\title{
Custos e padrões de tratamento de pacientes com câncer de pulmão não- pequenas células (CPNPC) avançado no sistema suplementar de saúde
}

\author{
Costs and treatment patterns in patients with advanced \\ non-small cell lung cancer in the private health system \\ Straus Tanaka', Thiago Chadid², Graziela Bernardino³ \\ DOI: 10.21115/JBES.v8.n2.p149-154
}

\section{RESUMO}

\section{Palavras-chave:}

neoplasia pulmonar, câncer de pulmão não-pequenas células, padrões de tratamento, custos, saúde suplementar

\section{Keywords:}

lung neoplasm, non-small cell lung cancer, treatment patterns, costs and supplementary health
Introdução: No Brasil, o câncer representa a segunda maior causa de morte, estando apenas atrás das doenças cardiovasculares e representando um grande impacto econômico observado pelo aumento no número de quimioterapias realizadas no país. Entre as neoplasias, o câncer de pulmão é o mais comum em todo o mundo e, de acordo com dados do INCA, em 2013 foi o câncer com o maior número de mortes. Objetivo: Estimar os custos médicos diretos do tratamento dos pacientes diagnosticados com câncer de pulmão não-pequenas células (CPNPC) no sistema de saúde suplementar. Método: Foi realizada uma análise retrospectiva em uma base de dados do sistema de saúde privado brasileiro entre o período de 2012 a 2015. Para o custeio de cada esquema terapêutico analisado foi utilizado o método de microcusteio em que os custos foram obtidos a partir da Classificação Brasileira Hierarquizada de Procedimentos Médicos, revista SIMPRO e lista CMED. Resultados: Foi demonstrado que $88 \%$ dos pacientes com câncer de pulmão são diagnosticados tardiamente (IIIB/IV) e possuem idade média de 65 anos, e apenas 10,15\% dos pacientes apresentaram resultados positivos para os testes genéticos de EGFR e ALK. Não foram observadas alterações na escolha terapêutica no período analisado e o custo de tratamento pode atingir $\mathrm{R} \$ 410.848,34$ no tratamento do paciente com CPNPC avançado. Conclusão: A análise demonstrou a necessidade crescente por terapias que promovam o aumento de sobrevida nos pacientes de CPNPC e que possam contribuir para otimizar os recursos utilizados.

\begin{abstract}
Introduction: In Brazil, the cancer represents the second cause of death, being only behind of cardiovascular diseases and representing a great economic impact, which is observed by the raise of chemotherapies performed in the country. Among neoplasm, lung cancer is the most common cancer around the world and in accordance with INCA data, in 2013, it was the cancer with the largest number of deaths. Objective: Estimate the direct medical costs of patients diagnosed with non-small cell lung cancer (NSCLC) in the private healthcare system. Methods: It was performed a retrospective analysis of diagnosed patients' data who were treated for advanced NSCLC during the period of 2012 to 2015 in the Brazilian private healthcare system. For the cost of each treatment regimen, it was used the micro-costing method in which the costs were obtained from Hierarchical Brazilian Classification of Medical Procedures, SIMPRO magazine, and CMED list. Results: The analysis demonstrated that $88 \%$ of patients with lung cancer are diagnosed late (IIIB/ IV) with the median
\end{abstract}

Recebido em: 06/07/2016. Aprovado para publicação em: 11/08/2016.

1. Analista, Departamento de Farmacoeconomia, Bristol-Myers Squibb, São Paulo, Brasil;

2. Gerente, Departamento Médico, Bristol-Myers Squibb, São Paulo, Brasil

3. Gerente, Departamento de Farmacoeconomia Bristol-Myers Squibb, São Paulo, Brasil.

Nome da instituição onde o trabalho foi executado: Bristol-Myers Squibb, São Paulo, Brasil

Informações sobre auxílios recebidos sob a forma de financiamento, equipamentos ou medicamentos: $O$ presente estudo recebeu suporte financeiro de Bristol-Myers Squibb, São Paulo, Brasil.

Contatos: Straus Tanaka, Rua Verbo Divino, 1.711 - Chácara Santo Antônio, São Paulo, SP, Brasil, CEP 04719-002, Tel.: +55 $113882-$ 2572, FAX: +55 11 3882-2024, e-mail: straus.tanaka@bms.com 
age of 65 years and only 10,15\% of patients had positive results for EGFR and ALK genetic tests. There were no observed changes in the therapeutic choice during the period analyzed and the cost of treatment can achieve R\$ 410.848,34 for the treatment of advanced NSCLC. Conclusion: The analysis demonstrated the increasing need of therapies that promote increasing survival in NSCLC patients and can contribute to optimize the resource use.

\section{Introdução}

O câncer é uma das principais causas de morbidade e mortalidade no mundo, o que torna esta doença um importante problema na gestão da saúde. E, embora as maiores taxas de incidência sejam encontradas em países desenvolvidos, mais de $60 \%$ das mortes relacionadas ao câncer ocorrem em países caracterizados por baixa ou média renda, onde os recursos para prevenção, diagnóstico e tratamento são limitados ou ausentes (Traebert et al., 2013).

No Brasil, o câncer representa a segunda maior causa de morte, ficando atrás apenas das doenças cardiovasculares, e representando um grande impacto econômico. De acordo com dados do INCA, entre os anos de 2011 e 2012, foi observado um aumento de $26 \%$ nos investimentos para o tratamento do câncer (de R\$ 1,9 bilhão para R\$ 2,4 bilhões). Estes dados refletem a informação do mapa assistencial da saúde de 2013, publicado pela Agência Nacional de Saúde Suplementar (ANS), em que é informado aumento de $47 \%$ de quimioterapias realizadas entre 2011 e 2012 (de 691.621 para 1.472.782), no sistema de saúde suplementar brasileiro (ANS, 2013).

E entre as neoplasias, o câncer de pulmão é o mais comum em todo o mundo, com 1,8 milhão de novos casos diagnosticados anualmente e com uma taxa de mortalidade de 1,6 milhão por ano (Brambilla et al., 2014). No Brasil, apenas em 2013, estima-se que o câncer de pulmão foi responsável por 24.490 mortes, representando naquele ano o câncer com o maior número de mortes (INCA-MS, 2014).

Uma medida utilizada pelo INCA para avaliar a gravidade da neoplasia é a observação da razão entre mortalidade e incidência, que mostra a relação entre o número de casos novos e o número de óbitos registrados em um determinado local e em período de tempo definido. Esta relação é consequência da sobrevida relativa média de cada tumor e é fortemente influenciada pelas características próprias de malignidade, possibilidade maior ou menor de um diagnóstico precoce e existência de tratamento eficaz (Mendonça et al., 2006).

Fica evidente a gravidade e a agressividade do câncer de pulmão quando observada a relação entre mortalidade e incidência deste câncer, demonstrando, desta forma, a necessidade de diagnósticos precoces e tratamentos que aumentem a sobrevida e a qualidade de vida destes pacientes.

\section{Objetivo}

Estimar os custos médicos diretos do tratamento dos pacientes diagnosticados com câncer de pulmão não-pequenas células (CPNPC) no sistema de saúde suplementar.

\section{Método}

Uma análise retrospectiva foi realizada a partir de uma base de dados de registro de pacientes com CPNPC avançado, identificados a partir do Código Internacional de Doenças (CID) referente ao câncer de pulmão e extraídos da base de dados administrativa Evidências - Kantar Health que cobre 3,8 milhões de vidas do mercado privado. Os seguintes CIDs foram selecionados:

- C34 Neoplasia dos brônquios e dos pulmões;

- C34.0 Neoplasia maligna do brônquio principal;

- C34.1 Neoplasia maligna do lobo superior, brônquio e pulmão;

- C34.2 Neoplasia maligna do lobo médio, brônquio ou pulmão;

- C34.3 Neoplasia maligna do lobo inferior, brônquio ou pulmão;

- C34.8 Neoplasia maligna dos brônquios e dos pulmões com lesão invasiva;

- C34.9 Neoplasia maligna dos brônquios ou pulmões, não especificado.

Foram incluídos indivíduos de ambos os sexos com diagnóstico de CPNPC, e que foram tratados em hospitais do sistema de saúde privado brasileiro, nos anos de 2012, 2013, 2014 e 2015. Para estes pacientes foram considerados os custos dos esquemas quimioterápicos realizados, custos com medicamentos, materiais utilizados para infusão/punção, honorários médicos e taxas de sala.

Para a definição dos materiais utilizados para a infusão de cada esquema posológico foi realizado um painel de especialistas referente ao tratamento de pacientes oncológicos, composto de oncologistas e enfermeiras oncológicas.

Para custear cada esquema de tratamento do CPNPC, foi utilizado o método de microcusteio que envolve a coleta de 
informações sobre o uso de recursos para cada intervenção (Frick, 2009).

A partir destes dados, os custos de exames, infusão e medicamentos foram ponderados pela porcentagem de utilização, a fim de obter o custo médio de cada tratamento. Também foram considerados nesta análise os custos com medicamento de suporte.

Os valores utilizados como fonte de custo para procedimentos e exames hospitalares foram retirados da tabela de Classificação Brasileira Hierarquizada de Procedimentos Médicos (CBHPM), 5a Edição. Para os materiais utilizados no procedimento de punção ou contraste foram utilizados os valores apresentados na revista SIMPRO - edição de outubro/ novembro de 2015 e, para medicamentos, os preços fábrica ICMS 18\% vigentes da lista CMED em novembro/2015.

Devido à baixa estabilidade dos medicamentos utilizados no tratamento do CPNPC não foi considerado o fracionamento de doses para a composição do custo.

\section{Resultados}

\section{Dados demográficos}

Do total de pacientes identificados na base de dados com câncer de pulmão ( $n=619)$, foram incluídos na análise apenas os pacientes com CPNPC metastáticos $(n=404)$.

A Figura 1 ilustra a análise da população elegível para o estudo e demonstra a estratificação destes pacientes por linhas de tratamento e manutenção terapêutica.
Do total de pacientes incluídos na análise, 57,9\% eram homens e 42,1\% mulheres; as demais características basais dos pacientes elegíveis estão descritas na Tabela 1.

\section{Diagnóstico}

\section{Mutações}

Referente aos exames diagnósticos, 15,84\% ( $n=64)$ da população incluída realizou exames genéticos para identificar mutações do gene EGFR $(n=60)$ ou rearranjos do gene ALK $(n=4)$, que são utilizados para auxiliar na seleção de pacientes que possam apresentar maior benefício com os inibidores de tirosiona quinase (TKIs) do EGFR e ALK (NCCN, 2014; Tseng et al., 2012).

Nenhum outro teste de mutação foi reportado.

Destes pacientes que realizaram os exames genéticos, 40 apresentaram resultados positivos para o gene EGFR e 1 para

Tabela 1. Características basais dos pacientes incluídos na análise

\begin{tabular}{lccc}
\hline & Média & Mediana & $\begin{array}{c}\text { Desvio } \\
\text { Padrão }\end{array}$ \\
\hline Idade (anos) & 64,62 & 65 & 12,54 \\
\hline Peso $(\mathrm{Kg})$ & 67,14 & 67 & 14,76 \\
\hline Altura $(\mathrm{m})$ & 1,65 & 1,65 & 0,13 \\
\hline Superfície corpórea $\left(\mathrm{m}^{2}\right)$ & 1,75 & 1,74 & 0,21 \\
\hline
\end{tabular}

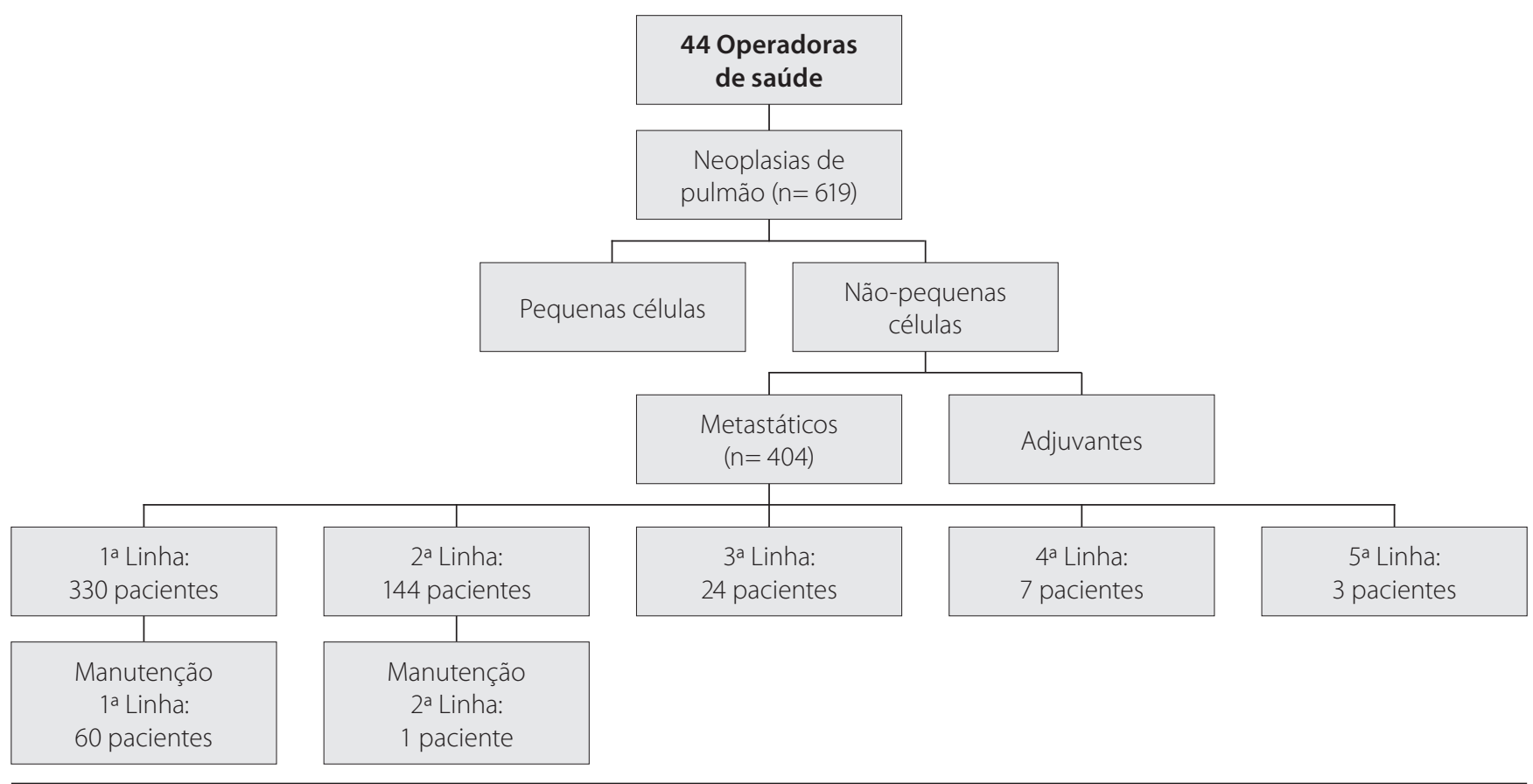

Figura 1. Análise da população elegível para o estudo 
o gene ALK, representando 9,9\% e 0,25\% do total de pacientes com CPNPC, respectivamente.

\section{Estadiamento}

O estadiamento do câncer é utilizado para determinar a extensão do câncer e onde ele está localizado, descrevendo a severidade da neoplasia baseado na magnitude do tumor primário (AJCC, 2016). Estes dados dependem do preenchimento correto das informações sobre o paciente ao diagnóstico e, assim, obtivemos informações registradas sobre o estadiamento do câncer de $63,86 \%$ ( $n=258$ ) dos pacientes. Destes pacientes, $87,98 \%(n=227)$ foram classificados como estádio IIIB/IV (avançado/metastático) e apenas 1,6\% em estádio II.

Tabela 2. Distribuição de pacientes por estadiamento do câncer

\begin{tabular}{lc}
\hline Estádio & $\mathbf{N}$ \\
\hline$\|$ & 2 \\
\hline$\| \mathrm{A}$ & 1 \\
\hline$\| \mathrm{b}$ & 1 \\
\hline$\| I$ & 20 \\
\hline$I \| \mathrm{A}$ & 7 \\
\hline$\| \mathrm{B}$ & 13 \\
\hline IV & 214 \\
\hline
\end{tabular}

Tabela 3. Locais de metástases registrados nos pacientes em análise

\begin{tabular}{ll}
\hline Local de metástase & N \\
\hline Óssea & 135 \\
\hline Linfonodo & 95 \\
\hline Fígado & 49 \\
\hline Pleura & 48 \\
\hline SNC & 52 \\
\hline Pulmão contralateral & 43 \\
\hline Adrenal & 27 \\
\hline Mediastino & 10 \\
\hline Parede torácica & 2 \\
\hline Pericárdio & 2 \\
\hline Cauda pâncreas & 1 \\
\hline Intestino delgado & 1 \\
\hline Olhos & 1 \\
\hline Paratraqueal & 1 \\
\hline Partes moles & 1 \\
\hline Pele & 1 \\
\hline Peritônio & 1 \\
\hline Rim & 1 \\
\hline Ventrículo E & 1 \\
\hline
\end{tabular}

\section{Metástases}

Os locais mais comuns de metástases foram ossos (28,6\%), nódulos linfáticos (20,1\%) e hepático (10,4\%). Na Tabela 3 estão listados todos os locais de metástases registrados nos pacientes em análise.

\section{Padrões de tratamento e custos}

Foi realizada uma análise individualizada por ano dos dados encontrados por linha terapêutica, durante o período de análise (2012 a 2015) a fim de identificar mudanças no padrão de tratamento. Para a análise do custo médio de pacientes com CPNPC em cada linha de tratamento foram considerados custos com os esquemas quimioterápicos (materiais, medicamentos, honorários e taxas), exames realizados, medicação de suporte e antieméticos.

\section{Primeira Linha}

Foram encontrados 330 pacientes com registros de terapias em primeira linha e 24 regimes quimioterápicos diferentes. 0 regime mais utilizado foi carboplatina com paclitaxel, 25,8\% $(n=85)$, seguido de carboplatina com pemetrexede, $24,2 \%$ $(n=80)$, e $18,8 \%(n=62)$ utilizaram regimes contendo bevacizumabe. $O$ uso de quimioterapia oral (erlotinibe e gefitinibe) foi de 7,0\% ( $n=23)$. A média de ciclos dos 24 regimes quimioterápicos utilizados foi de 4,28 ciclos.

$\mathrm{Na}$ análise individualizada por ano, apesar de observado aumento no número de opções de regimes quimioterápicos diferentes utilizados, houve poucas alterações no padrão de tratamento de CPNPC no decorrer do período estabelecido do estudo (2012 a 2015), predominando em todos os anos o uso do regime de carboplatina com paclitaxel e carboplatina com pemetrexede.

O custo do regime quimioterápico de primeira linha variou de $\mathrm{R} \$$ 4.914,39 (Cisplatina + Etoposídeo) a $\mathrm{R} \$ 132.785,45$ (Cisplatina + Pemetrexede + Bevacizumabe), sendo o custo médio ponderado pela porcentagem de utilização de

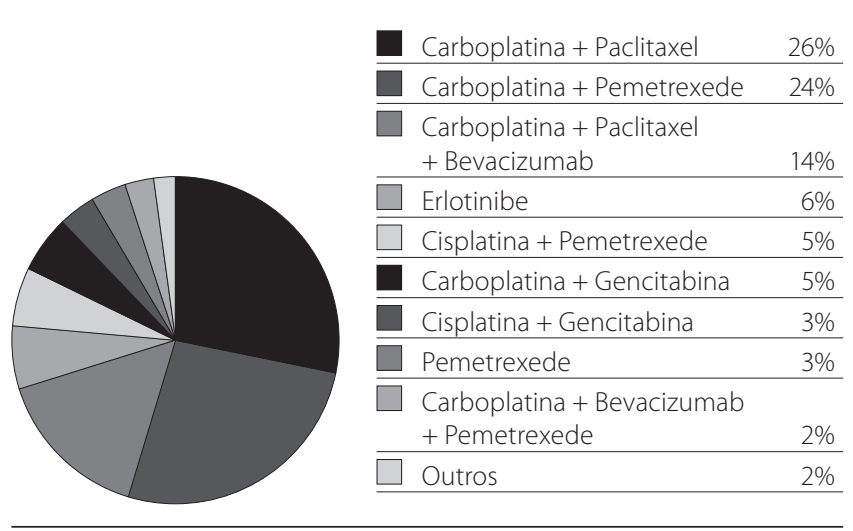

Figura 2. Regimes quimioterápicos utilizados no tratamento de primeira linha - todos os anos 
R\$ 46.293,93 (equivalente a 87,93\% do custo médio total). Os custos com antieméticos, exames e medicação de suporte somam $\mathrm{R} \$ 6.355,08$, e o custo médio total foi de $\mathrm{R} \$ 52.649,01$.

\section{Manutenção após primeira linha}

Foram encontrados 60 pacientes com registro de terapias de manutenção após a primeira linha e 4 regimes quimioterápicos diferentes. O regime mais utilizado foi pemetrexede em monoterapia, $66,67 \%(n=40)$. A média de ciclos dos 4 regimes quimioterápicos utilizados foi de 6,46 ciclos.

O custo do regime quimioterápico para manutenção após primeira linha variou de $\mathrm{R} \$ 14.744,21$ (gencitabina) a R\$ 151.243,28 (Pemetrexede + Bevacizumabe), sendo o custo médio ponderado pela porcentagem de utilização de R\$ 91.232,52 (equivalente a 93,34\% do custo médio total). Os custos com antieméticos, exames e medicação de suporte somam $R \$$ 6.504,78, e o custo médio total foi de R\$ 97.737,30.

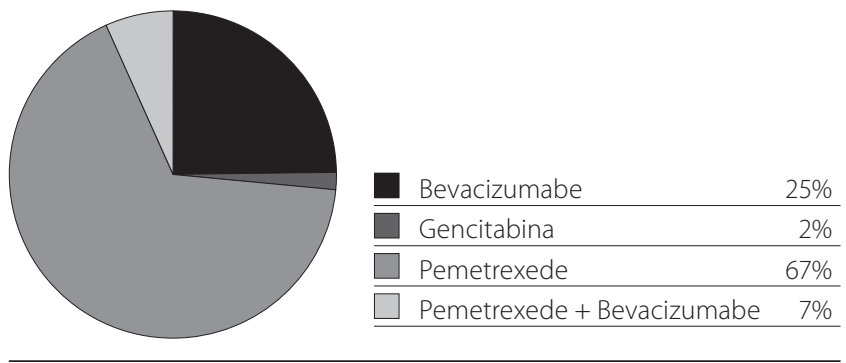

Figura 3. Regimes quimioterápicos utilizados no tratamento na manutenção após primeira linha

\section{Segunda linha e manutenção após segunda linha}

Foram encontrados 144 pacientes com registro de terapias em segunda linha e 10 regimes quimioterápicos diferentes. O regime mais utilizado foi docetaxel em monoterapia, 52,1\% $(n=75)$, seguido de pemetrexede em monoterapia, 27,8\% $(n=40)$. A média de ciclos dos 10 regimes quimioterápicos utilizados foi de 6,45 ciclos.

$\mathrm{Na}$ análise individualizada por ano referente às terapias em segunda linha, também foi observado o aumento no número de regimes quimioterápicos diferentes utilizados, mas com poucas alterações no padrão de tratamento ao longo

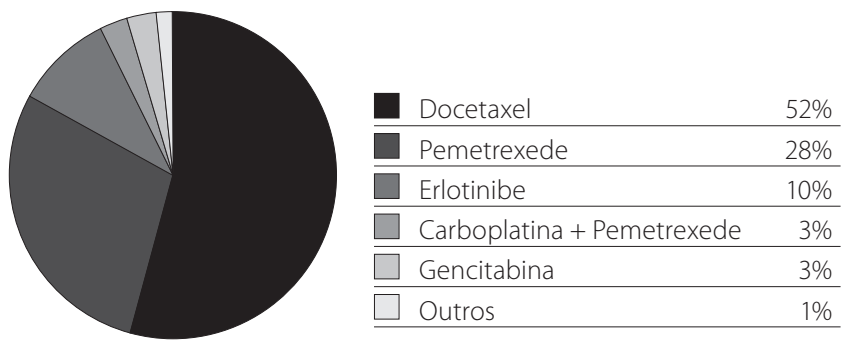

Figura 4. Regimes quimioterápicos utilizados no tratamento de segunda linha - todos os anos do período. Houve predomínio em todos os anos de monoterapias de docetaxel e pemetrexede.

O custo do regime quimioterápico de segunda linha variou de $\mathrm{R} \$$ 13.915,79 (erlotinibe) a $\mathrm{R} \$$ 106.630,37 (Carboplatina + Paclitaxel + Bevacizumabe), sendo o custo médio ponderado pela porcentagem de utilização de $R \$ 44.777,44$ (representando $89,18 \%$ do custo médio total). Os custos com antieméticos, exames e medicação de suporte somam R\$ 5.434.44, e o custo médio total foi de R\$ 50.211,88.

Para a manutenção após segunda linha foi encontrado apenas 1 paciente com uso de pemetrexede em monoterapia com registro de 11,5 ciclos. O custo total com antieméticos, exames e medicação de suporte deste tratamento foi de $\mathrm{R} \$ 132.720,48$.

\section{Demais Linhas}

Foram encontrados 34 registros de terapias nas demais linhas (terceira, quarta e quinta linha de tratamento) e 9 regimes quimioterápicos diferentes. O regime mais utilizado foi Erlotinibe, 52,9\% ( $n=18)$, seguido de docetaxel, $11,8 \%(n=4)$. A média de ciclos dos 9 regimes quimioterápico utilizados foi de 4,0 ciclos.

Não foi realizada a análise individualizada por ano nas demais linhas de tratamento devido ao baixo número de pacientes.

O custo do regime quimioterápico para as demais linhas de tratamento variou de $\mathrm{R} \$ 3.310,53$ (etoposideo) a $\mathrm{R} \$$ 87.805,87 (pemetrexede), sendo o custo médio ponderado pela porcentagem de utilização de R\$ 22.075,62 (representando $85,42 \%$ do custo médio total), e o custo médio total de R\$ 25.843.22.

\section{Uso de recursos (exames, antieméticos, medicamentos de suporte)}

Os exames mais realizados pelos pacientes elegíveis ao tratamento de CPNPC foram a tomografia computadorizada de tórax, 39,2\% ( $n=413)$, seguida da tomografia computadorizada de abdome total, 19,8\% ( $n=208)$, e PET CT, 17,0\% ( $n=179)$, correspondendo juntos a 76,0\% de todos os exames realizados.

Os antieméticos utilizados com maior frequência foram ondansetrona, seguido de granisetona e palonosetrona, e foi

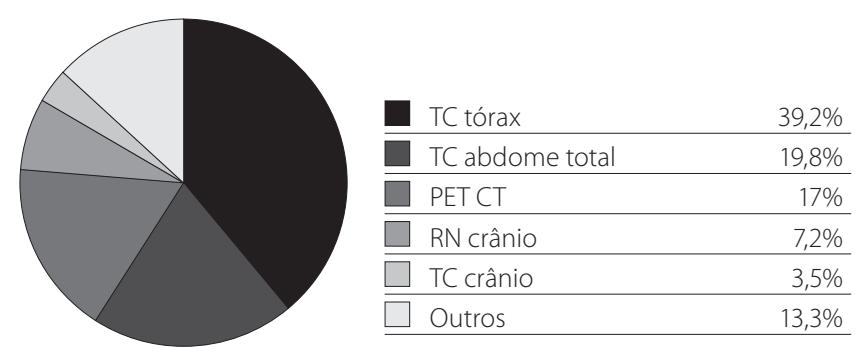

Figura 5. Recursos utilizados no tratamento de CPNPC 
observado maior uso de antieméticos em pacientes de primeira linha, $72,8 \%(n=210)$, em relação às linhas subsequentes. Entre os medicamentos de suporte, o mais utilizado foi o ácido zoledrônico, 88,6\% ( $n=109)$, seguido de eritropoietina, $6,5 \%(n=8)$, e filgrastina, 3,3\% ( $n=4)$.

Considerando todas as unidades de custo avaliadas (medicamentos quimioterápicos, materiais, honorários e taxas, medicamentos de suporte, medicamentos antieméticos e exames) os custos médios obtidos por linha de tratamento estão representados na Tabela 4.

Tabela 4. Custo médio total por linha de tratamento

\begin{tabular}{lc}
\hline Linha de tratamento & $\begin{array}{c}\text { Custo médio } \\
\text { total }\end{array}$ \\
\hline Primeira linha de tratamento & $\mathrm{R} \$ 52.649,01$ \\
\hline Manutenção após primeira linha & $\mathrm{R} \$ 97.737,91$ \\
\hline Segunda linha de tratamento & $\mathrm{R} \$ 50.211,88$ \\
\hline Manutenção após segunda linha & $\mathrm{R} \$ 132.720,48$ \\
\hline Terceira linha de tratamento & $\mathrm{R} \$ 29.121,08$ \\
\hline Quarta linha de tratamento & $\mathrm{R} \$ 33.786,35$ \\
\hline Quinta linha de tratamento & $\mathrm{R} \$ 14.622,22$ \\
\hline
\end{tabular}

\section{Discussão}

Os dados demográficos mostram que a população brasileira com câncer de pulmão é caracterizada por pacientes idosos (idade média de 65 anos). Apenas 10,15\% apresentaram resultados positivos para os testes genéticos de EGFR e ALK, e quase $88 \%$ dos pacientes com resultados de estadiamento são diagnosticados tardiamente com a doença em fases avançadas (estádios IIIB/IV).

Em relação ao padrão de tratamento de CPNPC, apesar do aumento da diversificação de regimes quimioterápicos utilizados ao longo do intervalo de análise (2012 a 2015), não foram observadas grandes mudanças na escolha terapêutica. Para a primeira linha terapêutica, os regimes mais utilizados em todos os anos foram a carboplatina com paclitaxel e carboplatina com pemetrexede, que juntos foram responsáveis por 50\% dos tratamentos registrados; para a segunda linha houve predomínio em todos os anos de docetaxel com 52,1\%; e para as demais linhas houve preferência por erlotinibe com $52,9 \%$.

Estes dados evidenciam a ausência de novas terapias que apresentem benefício clínico adicional significativo em relação aos padrões de tratamento já estabelecidos.

Adicionalmente, também foi avaliado o custo de tratamento, que se mostrou bastante significativo, podendo atingir $\mathrm{R} \$ 410.848,34$ em um paciente que permaneça em tratamento passando por todas as linhas terapêuticas disponíveis.
Essa análise confirma o resultado de um estudo que coletou dados sobre as taxas de sobrevida em 5 anos medidas em dois períodos: de 1974 a 1976 e de 2002 a 2008, no qual foi confirmado que a evolução na expectativa de sobrevida do câncer de pulmão foi bastante inferior quando comparada a outros tipos de câncer com alta incidência (Lung Cancer Alliance, 2013). Assim, há uma necessidade por tratamentos que tragam maior sobrevida de longo prazo aos pacientes de CPNPC em relação aos tratamentos atualmente disponíveis, e que possam contribuir desta forma para a redução do número de mortes registradas no Brasil e no mundo.

\section{Conclusão}

Devido à escassez de dados epidemiológicos brasileiros, esta análise se mostra útil no conhecimento dos padrões de tratamento utilizados, servindo como uma ferramenta fundamental para formuladores de políticas de saúde e gestores, no processo decisório.

O resultado desta análise demonstra que o tratamento do câncer de pulmão pode acarretar um impacto financeiro significativo no sistema privado de saúde. A doença apresenta um alto custo e possui alta letalidade devido ao seu diagnóstico tardio.

Assim, frente ao custo elevado demonstrado na análise, e em um cenário onde os recursos são escassos e limitados, há uma necessidade crescente por terapias que busquem otimizar os recursos utilizados e promovam o aumento de sobrevida no tratamento de CPNPC.

\section{Referências bibliográficas}

Agência Nacional de Saúde Suplementar (ANS). Mapa assistencial de saúde suplementar, 2013.

Brambilla E, Travis WD. Lung cancer. In: World Cancer Report, Stewart BW, Wild CP (Eds). World Health Organization, Lyon 2014.

Frick KD. Microcosting quantity data collection methods. Med Care, v. 47, n. 7 Suppl 1, p. S76-81, 2009.

INCA-MS. Estimativa - Incidência de Câncer no Brasil. Rio de Janeiro: Ministério da Saúde. Secretaria de Atenção à Saúde. Instituto Nacional de Câncer. Coordenação de Prevenção e Vigilância, 2014.

Lung Cancer Alliance. Lung Cancer Facts. Disponível online em: http:// www.lungcanceralliance.org/assets/docs/media/LCA\%20Funding\%20 Fact\%20Sheet\%202012.pdf. Acessado em 5 de Fevereiro de 2016.

Mendonça GAS, Noronha CP, Almeida LM. A situação do câncer no Brasil (Instituto Nacional de Câncer - INCA), 2006.

National Comprehensive Cancer Network (NCCN). Clinical practice guidelines in oncology: Non-small cell lung cancer. Version 2. 2014.

Traebert J, Schneider IJ, Colussi CF, De lacerda JT. Burden of disease due to cancer in a Southern Brazilian state. Cancer Epidemiol, v. 37, n. 6, p. 788-792, 2013.

Tseng JS, Yang TY, Chen KC, Hsu KH, Chen HY, Chang GC. Retrospective study of erlotinib in patients with advanced squamous lung cancer. Lung Cancer, v. 77, n. 1, p. 128-133, 2012. 